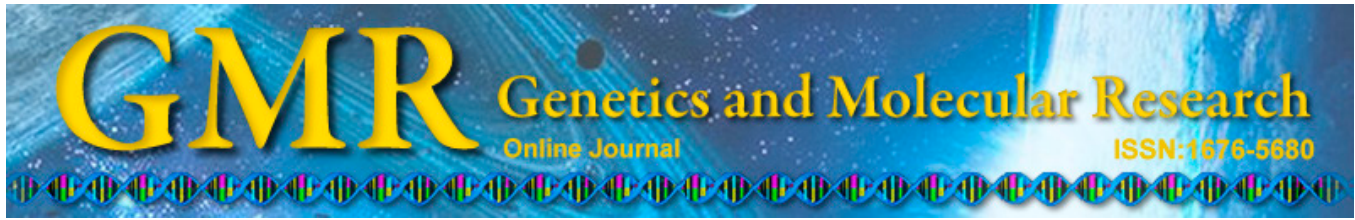

\title{
Transcriptional responses in eastern honeybees (Apis cerana) infected with mites, Varroa destructor
}

\author{
T. Ji', L. Yin ${ }^{2}$, Z. Liu ${ }^{1,3}$, Q. Liang ${ }^{4}$, Y. Luo ${ }^{5}$, J. Shen ${ }^{1}$ and F. Shen ${ }^{1}$ \\ ${ }^{1}$ College of Animal Science and Technology, Yangzhou University, \\ Yangzhou, China \\ ${ }^{2}$ Institute of Food Science and Technology, \\ Jiangsu Agri-Animal Husbandry Vocational College, Taizhou, China \\ ${ }^{3}$ Shandong Apicultural Association, Jinan, China \\ ${ }^{4}$ College of Bee Science, Fujian Agriculture and Forestry University, \\ Fuzhou, China \\ ${ }^{5}$ Guangdong Entomological Institute, Guangzhou, China \\ Corresponding author: $\mathrm{T}$. Ji \\ E-mail: tji@yzu.edu.cn
}

Genet. Mol. Res. 13 (4): 8888-8900 (2014)

Received March 13, 2013

Accepted August 22, 2014

Published October 31, 2014

DOI http://dx.doi.org/10.4238/2014.October.31.4

\begin{abstract}
The Varroa destructor mite has become the greatest threat to Apis mellifera health worldwide, but rarely causes serious damage to its native host Apis cerana. Understanding the resistance mechanisms of eastern bees against Varroa mites will help researchers determine how to protect other species from this organism. The A. cerana genome has not been previously sequenced; hence, here we sequenced the $A$. cerana nurse workers transcriptome and monitored the differential gene expression of $A$. cerana bees challenged by $V$. destructor. Using de novo transcriptome assembly, we obtained 91,172 unigenes (transcripts) for A. cerana. Differences in gene expression levels between the unchallenged (Con) and challenged (Con2) samples were estimated, and a total of 36,691 transcripts showed a 2-fold difference (at least)
\end{abstract}


between the 2 libraries. A total of 272 differentially expressed genes showed differences greater than 15 -fold, and 265 unigenes were present at higher levels in Con 2 than in Con. Among the upregulated unigenes in the Con 2 colony, genes related to skeletal muscle movement (troponin and calcium-transporting ATPase), olfactory sensitivity (odorant binding proteins, and Down syndrome cell adhesion molecule gene) and transcription factors (cyclic adenosine monophosphate-responsive element-binding protein and transcription factor mblk-1) appeared to be involved in Varroa resistance. Real-time polymerase chain reaction was performed to validate these differentially expressed genes screened by the sequencing approach, and sufficient consistency was observed between the two methods. These findings strongly support that hygienic and grooming behaviors play important roles in Varroa resistance.

Key words: Apis cerana; Gene expression; Transcriptome; Varroa destructor

\section{INTRODUCTION}

Varroa destructor is a parasitic mite that damages bees both physically and physiologically by feeding on the hem lymph of developing and adult bees (Rosenkranz et al., 2009). Importantly, the mite also serves as a vector and inducer of several highly pathogenic honeybee viruses, causing severe damage to bee colonies and often leading to colony death (Annoscia et al., 2012). Numerous honeybee viruses are associated with Varroa mite infestation, most notoriously colony collapse disorder (Highfield et al., 2009; Cornman et al., 2012) and deformed wing virus (Prisco et al., 2012).

$V$. destructor switched from its native host from Apis cerana to Apis mellifera 4 decades ago, later spreading to nearly all parts of the world (Sammataro et al., 2000) and becoming the greatest threat to A. mellifera health worldwide. The need for regular mite control treatments (typically chemical acaricides) to control the parasite population in western honeybee apiculture efforts (Annoscia et al., 2012) resulted in the rapid evolution of mite resistance against these chemicals, decreasing their efficiency (Damiani et al., 2009; Kanga et al., 2010). These control treatments often contaminate apicultural products (honey, pollen) with acaricide residues (Karazafiris et al., 2008). As such, alternative strategies are needed to fight $V$. destructor that will neither facilitate parasite resistance nor contaminate bee products (Behrens et al., 2012). Breeding Varroa-resistant honeybees is an ideal strategy, as it either reduces or eliminates the need for acaricides without requiring additional Varroa control measures. Vast resources and attention have been invested in the breeding of $V$. destructor-resistant $A$. mellifera (Rinderer et al., 2010) by searching for quantitative trait loci and identifying Varroaresistant honeybee genes.

Although A. mellifera are susceptible to these mites of Asian origin, frequently resulting in colony disintegration, the mites rarely cause serious damage to their native hosts, A. cerana (Oldroyd, 1999). In fact, in some locations in China, it is difficult to find even a single Varroa mite within an A. cerana population. Natural selection for benign host-parasite interactions may play a role in these relationships, allowing for co-existence between eastern bees and mites. Several factors have been implicated in this interaction, including grooming 
and hygienic behavior and differences in developmental timing. The mechanisms underlying the genetic differences in the eastern Varroa-resistant bees remain unclear. Quantitative trait loci mapping techniques have been used to study gene expression, identify resistance genes (Parker et al., 2012), and identify chromosomal regions that contain genes influencing specific traits in resistant and susceptible A. mellifera lines (Behrens et al., 2012; Tsuruda et al., 2012). However, these studies used different breeding lines. As such, candidate genes and quantitative trait loci were difficult to confirm in other studies. Furthermore, Varroa resistance is likely regulated by several genes and markers, and A. cerana is an aggregate of several resistance characteristics. Rath and Drescher (1990) found that it is necessary to thoroughly clarify the relationship between Varroa and A. cerana before the relationship between Varroa and A. mellifera can be discerned.

To identify essential genes involved in the response of honeybees to Varroa infection, we utilized the Illumina Solexa sequencing method to determine the head transcriptome of A. cerana worker bees and monitor their expression transcripts after Varroa infection. The objective of this study was to identify genes likely to be involved in $V$. destructor resistance and ultimately provide information that can be used to develop an environmentally friendly method for protecting A. mellifera from this serious parasite.

\section{MATERIAL AND METHODS}

\section{Honeybee colonies and Varroa challenge}

The A. cerana population was bred in the Guangdong Entomological Institute, Guangzhou, China. We selected a piece of honeycomb from an infested brood within an A. mellifera colony containing mature female mites in worker brood cells and several capped worker brood cells and capped drone brood cells (Tan et al., 2002). The comb was then introduced into the A. cerana colony. Nurse workers of the A. cerana colony were collected both before and after 24-h challenge. Additionally, nurse workers exhibited marked grooming and removal of Varroa mites. Control treatments were applied, which included non-parasitized samples referred to as Con, while parasitized samples were referred to as Con2. To assess the grooming behavior and removal rate, a white sheet was placed onto the hive bottom of the former 4 colonies to count the dead mites killed by A. cerana nurse bees (Figure 1).

\section{Sibling estimation by microsatellite genotyping}

To minimize the effect of genetic variation, pools of full-sister workers (related by $75 \%$ due to haplodiploidy) were collected in equal numbers from the 2 colonies (Navajas et al., 2008). To identify full sisters, we genotyped 100 bees from each colony using the Ap53 and A107 microsatellite loci (Franck et al., 1999).

DNA was extracted from the thorax, amplified by polymerase chain reaction (PCR), and scored on an $8 \%$ polyacrylamide gel using an ABI 377 automated DNA analyzer (Applied Biosystems, Foster City, CA, USA). Electropherogram processing was performed using the GENESCAN3.1 software (Applied Biosystems), and allele-sized scoring was conducted using the Binthere software (Applied Biosystems). The genotype data were used to assemble sets of 10 workers belonging to the same full sibling group. This procedure minimizes the effects of intracolonial genetic variation on gene expression. 


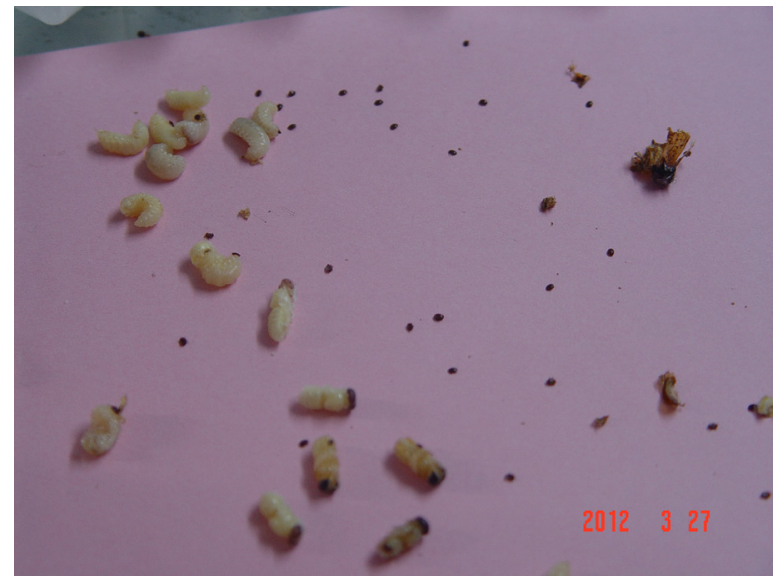

Figure 1. Assessment of grooming behavior and removal rate. A white sheet was placed onto the hive bottom to count the dead mites after 24-h infection by Varroa mites.

\section{RNA extraction, library preparation, and sequencing}

Total RNA was extracted from the head of the samples (each pooled from 30 honey bees) using TRIzol reagent (Invitrogen, Carlsbad, CA, USA). A Qubit fluorometer (Invitrogen) and an Agilent 2100 Bioanalyzer (Agilent Technologies, Inc., Santa Clara, CA, USA) were used to determine the quality and quantity of RNA. The mRNA was enriched using oligo(dT) magnetic beads, then broken into short fragments (approximately 200-700 nt) using fragmentation buffer (Invitrogen). For first-strand cDNA synthesis, the mRNA fragments were used as the template, and a random hexamer primer was used according to manufacturer instructions. Following second-strand synthesis, the double-stranded cDNA product was purified using the QiaQuick PCR extraction kit (Qiagen, Hilden, Germany) and washed with EB buffer for end repair and poly(A) addition. Finally, sequencing adapters were ligated to the fragments, and the fragments were purified by agarose gel electrophoresis and enriched by PCR amplification. The library products were sequenced using the Illumina HiSeq ${ }^{\mathrm{TM}} 2000$ system (San Diego, CA, USA).

\section{De novo transcriptome assembly and analysis of transcriptome sequencing results}

The de novo transcriptome assembly process was conducted using the Trinity short reads assembly program (Grabherr and Haas, 2011). Functional annotation information was obtained from Clusters of Orthologous Groups (COGs) and Gene Ontology (GO) for the unigenes. Unigene sequences were first aligned using BLASTX to protein databases, such as nr, Swiss-Prot, KEGG, and COGs (e-value $<0.00001$ ) and then aligned using BLASTn to nucleotide databases nt (e-value $<0.00001)$ to retrieve proteins with the highest sequence similarity to the unigenes along with their protein functional annotations.

\section{Differentially expressed gene (DEG) identification}

The unigene expression calculation uses the reads per kb per million reads method 
(Mortazavi and Williams, 2008), and expression between two samples can be calculated using the false discovery rate (FDR) method. The FDR method has been used to determine the P value threshold in multiple tests and analyses (Benjamini and Yekutieli, 2001). In our analysis, we considered genes with FDR $\leq 0.001$ and a ratio $>2$ to be significantly differentially expressed. DEGs were then analyzed using GO functional analyses and KEGG pathway analyses.

Different genes typically work cooperatively to exercise their biological functions. Pathway-based analysis helped to identify a gene's biological functions. Pathway enrichment analysis using public pathway-related databases, such as KEGG, were used to identify significantly enriched metabolic pathways or signal transduction pathways in DEGs compared with the entire genome. This pathway enrichment method was used to determine the main biochemical and signal transduction pathways in which the DEGs participate.

\section{DEG quantification using real-time quantitative PCR (RT-qPCR)}

The results of high throughput sequencing were confirmed by measuring the expression of 8 differentially regulated genes with potential roles in Varroa resistance using RTqPCR. These analyses were carried out using unchallenged (Con) and challenged (Con2) samples. The $t$-test was carried out to analyze the differentiation significance of mRNA expression between Con and Con2 using the SPSS 11.0 software (SPSS, Inc., Chicago, IL, USA). Primer sequences for the 8 target genes and the internal control gene (actin) are listed in Table 1.

\begin{tabular}{lll}
\multicolumn{1}{c}{ Table 1. Primers for target genes. } & & \\
\hline Target gene & Upstream primer $\left(5^{\prime}-3^{\prime}\right)$ & Downstream primer $\left(5^{\prime}-3^{\prime}\right)$ \\
\hline Troponin & GGCTGCACACTCTCTTCCTC & TCCCAATCCGAATGAATGAT \\
Calcium-transporting ATPase & GCGTCATTTACACCATCGCC & CGACGATCTTCCTCCGTCTG \\
Odorant binding protein4 & CGTCTTCGTCTGCAGCTTTG & AACACCCGACGACTTTACGG \\
Odorant binding protein17 & TGTTGAGCAGTGCCAATTC & TGCTATTTGCGTTGGGTA \\
Odorant binding protein18 & TCAGCATCAGAGATAGCCGAAC & TGGCTATGACGATGGTGGAAA \\
$\begin{array}{l}\text { Down syndrome cell adhesion molecule gene } \\
\text { cAMP-responsive element-binding protein-like 2-like }\end{array}$ & GCTGTACTCGTGCAGGTCT & ACGTGTCTTCGTGTCGAGTC \\
Transcription factor mblk-1-like & CAGCAATCGTAAGGTGGGGT & GTTGCCCTCGTATTCCCGAT \\
Actin & GGGGAATCTTAAAGGCGAAG & TGCCAAGCACTACACCAAAA \\
\hline
\end{tabular}

\section{RESULTS}

\section{Sequencing and assembly}

Two pools of mRNA samples (each from Con and Con2) were used to build libraries for the high-throughput parallel sequencing process using Illumina sequencing technology. After conducting cleaning and quality checks, we obtained 49,380,841 and 51,268,675 clean reads for Con and Con2, respectively. All high-quality reads were assembled de novo using the Trinity program, first into contigs and then using sequence overlap information until the contigs were no longer extended. This assembly produced 74,891 and 116,013 contigs from Con and Con2, with average lengths of 512 and 277 base pairs (bp) and N50s (N50 represents median length of all contigs) of 1448 and $373 \mathrm{bp}$, respectively. Using the paired-end joining and gap-filling techniques, the contigs were further assembled into unigenes that could not be extended on either ends. We obtained a total of 70,620 and 61,815 unigenes from Con and 
Con2, respectively, with final unigene N50 lengths of 3458 and $1090 \mathrm{bp}$, respectively. We generated 91,172 all-unigene sequences from both samples with an average length of 1858 bp, a final all-unigene sequence N50 length of $3774 \mathrm{bp}$ and a total length of 169,402,078 bp.

\section{Functional transcriptome annotation}

Because genomic information for $A$. cerana was lacking, we performed BLAST alignments (e-value $<1.00 \mathrm{E}-5$ ) against the NCBI NR, NT, SwissProt, GO, and KEGG databases to identify the putative functions of the all-unigene sequences. A total of 63,496 all-unigenes $(69.6 \%$ of all) matched 1 or more of the databases, more than half of the unigenes $(55.04 \%)$ matched the western bee (A. mellifera) from the $\mathrm{nr}$ database, and 26.57\% matched Apis florea (Table 2).

Table 2. Species distribution of the BLASTX results against the nr database.

\begin{tabular}{lrr}
\hline Species & Gene No. & Percentage \\
\hline Apis mellifera & 28,486 & $55.04 \%$ \\
Apis florea & 13,752 & $26.57 \%$ \\
Bombus impatiens & 2362 & $4.56 \%$ \\
Bombus terrestris & 2190 & $4.23 \%$ \\
Megachile rotundata & 1049 & $2.03 \%$ \\
Harpegnathos saltator & 480 & $0.93 \%$ \\
Other & 3438 & $6.64 \%$ \\
\hline
\end{tabular}

According to GO classifications, 85,577 unigenes were categorized into biological process $(85,577$ members), cellular component (48,194 members), and molecular function (29,301 members) (Figure 2). Most cellular components were categorized into cell (10,323; $21.42 \%)$ and cell parts $(10,323 ; 21.42 \%)$. Within the molecular function category, binding $(11,370 ; 38.80 \%)$ and catalytic $(11,042 ; 37.68 \%)$ activities were dominant. Within the biological process category, cellular $(14,797 ; 17.29 \%)$ and metabolic $(10,371 ; 12.12 \%)$ processes were the most highly represented terms.

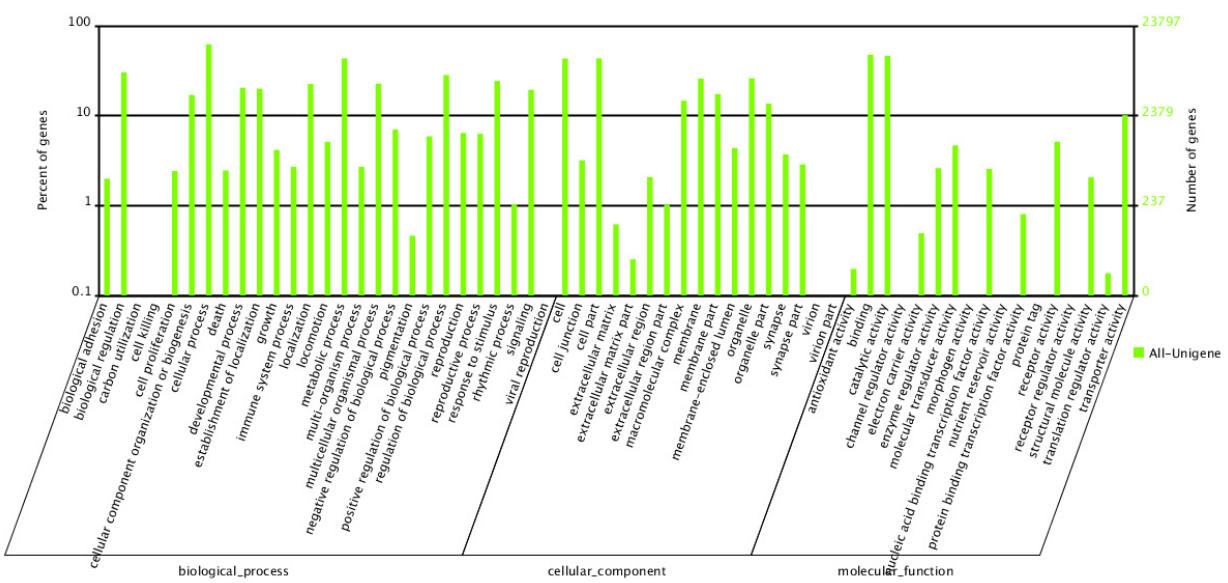

Figure 2. Histogram of the Gene Ontology database classification of all unigenes. The GO terms were classified into 3 categories from the GO database, including biological processes, molecular functions, and cellular components on the X-axis. The percent and number of genes are shown on the two Y-axes. A total of 85,577 unigenes were categorized into biological process, 48,194 into cellular component, and 29,301 into molecular function. 
We also searched annotated sequences for genes involved in COG classifications. A total of 23,790 sequences had a COG classification (Figure 3). Among the 25 COG categories, "general function prediction only" represented the largest group (10,447 members), followed by "replication, recombination, and repair" (4794 members), "transcription" (4203 members), "translation, ribosomal structure, and biogenesis" (4113 members), and "signal transduction mechanisms" (3362 members). The categories representing extracellular structures (37 members) and nuclear structures ( 25 members) were the smallest groups (Figure 3 ). We also mapped the annotated sequences to the reference canonical pathways in the KEGG database. We assigned a total of 39,625 sequences to 258 KEGG pathways. The pathways with the greatest representation of unique sequences were the metabolic pathways (4793 members), followed by regulation of the actin cytoskeleton ( 1650 members), those related to focal adhesion (1486 members), and pathways in cancer (1456 members) (data not shown).
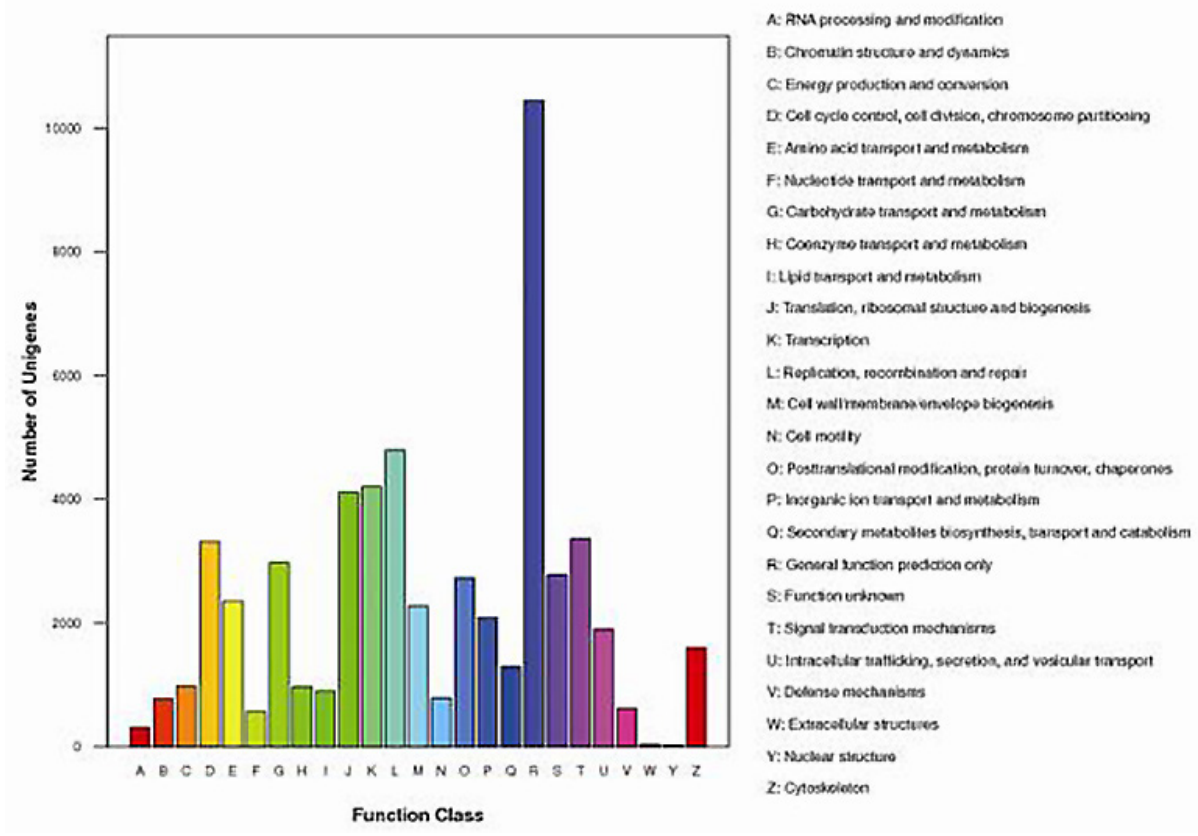

Figure 3. Clusters of Orthologous Groups of Proteins database classification of all unigenes by function. Designations of functional categories of unigenes in alphabetical order on $\mathrm{X}$-axis are shown for the given items according to the right side.

\section{DEGs in the Con and Con2}

Differences in gene expression levels between the Con and Con2 libraries were used to scan for genes associated with Varroa resistance. An FDR $<0.001$ and absolute value of $\mid \log 2$ Ratio $\mid \geq 1$ were used as the statistical significance threshold. Transcripts with at least a 2 -fold difference between the 2 libraries are shown in Figure 4. A total of 36,691 identified genes were differently expressed between Con and Con2. Of these, 17,799 were downregulated and 18,892 were upregulated in Con2 bees. 


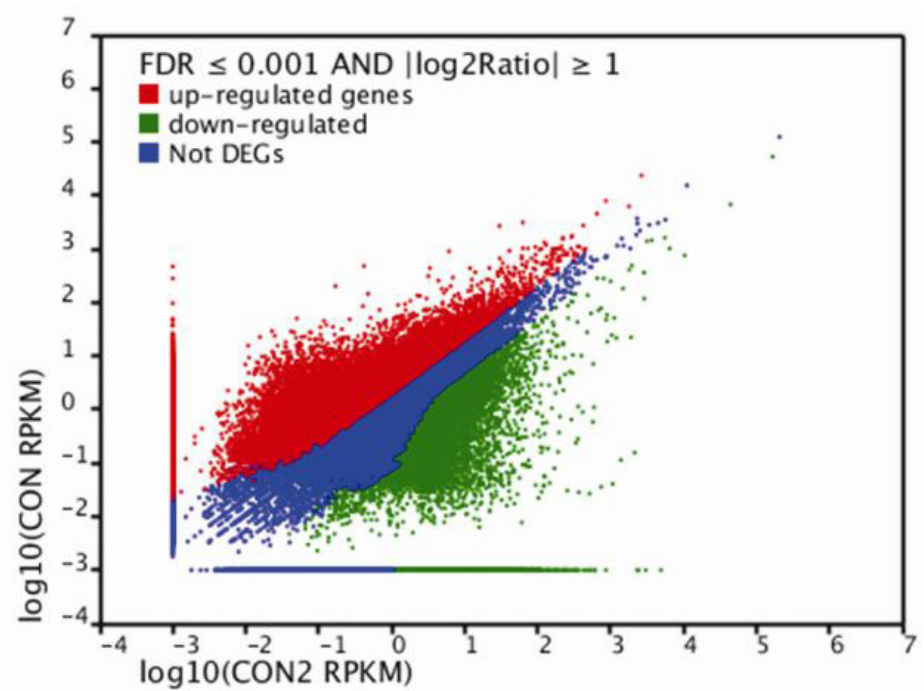

Figure 4. Comparison of gene expression levels between the Con and Con2 libraries. To compare gene expression levels, each library was normalized to 1 million tags. The red dots indicate transcripts that were more prevalent in the Con library, the green dots indicate transcripts that were more prevalent in the Con2 library, and the blue dots indicate transcripts that were similar in both libraries. A false discovery rate $\leq 0.001$ and a $\log _{2}$ ratio $\geq 1$ were used as the threshold of statistically significant gene expression differences.

\section{GO functional enrichment and KEGG pathway enrichment analyses}

We identified 6 significantly enriched GO terms in DEGs compared to the genome background and found that binding was the most significantly enriched GO term in the former. Other identified GO terms were related to structural molecular activity and structural ribosomal constituents (data not shown). Pathway enrichment analysis identified significantly enriched pathways in the DEGs by comparison with the whole genome background. We found that 258 pathways were affected by 5721 DEGs; of these, 50 pathways with a Q-value $\leq 0.05$ were significantly enriched. The most significantly enriched pathways included metabolic pathways (1528 DEGs), RNA transport (428 DEGs), and spliceosomes (328 DEGs) (Table S1).

\section{Highly expressed transcripts in Con and Con2 colonies}

A total of 272 DEGs with differences more than 15-fold and 265 unigenes were present at higher levels in the Con2 colony compared to the Con colony, while 7 were present at lower levels, suggesting that numerous additional genes were highly upregulated in the Con2 colony compared to the Con colony. Among the 7 downregulated unigenes in the Con colony, 3 were unknown and none of the remaining 4 appeared to be related to Varroa resistance. Among the 265 upregulated unigenes in the Con2 colony, several appeared to be involved in Varroa resistance, including genes related to skeletal muscles (troponin and calcium-transporting ATPase), olfactory sensitivity (odorant binding protein $4,17,18$, and Down syndrome cell adhesion molecule gene), and transcription factors [cyclic adenosine monophosphate (cAMP) responsive element-binding protein-like 2-like and transcription factor mblk-1-like (Mblk-1)] (Table 3). 
Table 3. Genes with potential roles in Varroa resistance.

\begin{tabular}{lccc}
\hline Gene & Fold by DEG & Fold by qRT-PCR & P value \\
\hline Troponin & 17.6908 & 115.3606 & 0.0235 \\
Calcium-transporting ATPase & 17.8542 & 8.9907 & 0.0336 \\
Odorant binding protein 4 & 15.8859 & 223.41 & 0.0319 \\
Odorant binding protein 17 & 17.3884 & 17.2313 & 0.0254 \\
Odorant binding protein 18 & 17.2985 & 10.3564 & 0.0391 \\
Down syndrome cell adhesion molecule gene & 13.7496 & 11.2815 & 0.0351 \\
cAMP-responsive element-binding protein-like 2-like & 16.8001 & 40.56 & 0.0219 \\
Transcription factor mblk-1-like & 16.0286 & 11.2565 & 0.036 \\
\hline
\end{tabular}

\section{Verification of DEGs}

To further evaluate the DEGs, 8 differentially regulated genes with potential roles in Varroa resistance were analyzed by RT-qPCR. Differentiation of expression of these 8 target genes between Con and Con2 were significant $(\mathrm{P}<0.05)$; the ratio of Con and Con2 are shown in Table 4. Consistent expression patterns were observed in transcriptome analysis, despite some quantitative differences in expression level. Their significant agreement indicated that the abundance of Illumina sequences from the A. cerana transcriptome represented the actual expression level.

Table 4. Comparisons of differentially expressed gene (DEG) data and RT-qPCR results.

\begin{tabular}{|c|c|c|c|}
\hline Gene & Fold by DEG & Fold by RT-qPCR & $P$ value \\
\hline Troponin & 17.6908 & 115.3606 & 0.0235 \\
\hline Calcium-transporting ATPase & 17.8542 & 8.9907 & 0.0336 \\
\hline Odorant binding protein 4 & 15.8859 & 223.41 & 0.0319 \\
\hline Odorant binding protein 17 & 17.3884 & 17.2313 & 0.0254 \\
\hline Odorant binding protein 18 & 17.2985 & 10.3564 & 0.0391 \\
\hline Down syndrome cell adhesion molecule gene & 13.7496 & 11.2815 & 0.0351 \\
\hline cAMP-responsive element-binding protein-like 2-like & 16.8001 & 40.56 & 0.0219 \\
\hline Transcription factor mblk-1-like & 16.0286 & 11.2565 & 0.036 \\
\hline
\end{tabular}

\section{DISCUSSION}

Numerous studies have shown that grooming and hygienic behaviors are the most important mechanisms of Varroa resistance in A. cerana. Grooming behavior has been measured in A. mellifera, but less frequently than in A. cerana (Bienefeld et al., 1999). Troponin and calcium-transporting adenosine triphosphatase (ATPase) were highly upregulated in Con2, indicating that troponin and calcium-transporting ATPase may play important roles in the grooming and hygienic behaviors.

Troponin is involved in striated muscle excitation contraction as the main regulatory protein. The troponin complex is composed of 3 subunits, including troponin $\mathrm{C}$, troponin I, and troponin $\mathrm{T}$, and mediates the thin-filament response to calcium when striated muscular contraction is initiated. In mammalian systems, extensive studies (O'Connell et al., 2008) have addressed the structure-function relationships between Tpn complex subunits. When $\mathrm{Ca}^{2+}$ binds to troponin $\mathrm{C}$, this polypeptide undergoes a conformational change to alter its interaction with troponin I, releasing inhibitory troponin I-actin binding, and allowing movement of the troponin-tropomyosin complex on the F-actin; this increases myosin's ability to bind to actin and thus promotes contraction. Studies in insects have focused on the function of tro- 
ponin within the muscles responsible for flight in the adult thorax (Herranz et al., 2005). These muscles, known as indirect flight muscles, are responsible for wing beating, while the tergal depressor of the trochanter muscle is responsible for a fly's ability to jump at take-off. The high troponin expression detected within the heads of bees from the Con 2 colony indicated that striated muscles within the heads of these bees contract and relax strongly upon Varroa infection.

In skeletal muscle, calcium-transporting ATPase can control muscle contraction and heat production, activities that signal the metabolism of various cells (Sørensen et al., 2004). The highly differential expression of calcium-transporting ATPase between the Con and Con2 colonies also suggests that $A$. cerana showed stronger muscle movements upon Varroa infection. There are also reports of mites that were injured by the mandibles of worker bees (Rosenkranz et al., 1997). A. cerana worker bees (nurse-age worker bees) can also detect capped broods infested by $V$. destructor; in such cases, the bees open the infested cells to remove the mites, a phenomenon known as hygienic or removal behavior (Boecking, 1992). Both of these behaviors require the use of the muscles in the head, particularly in the mandibles, so high expression of troponin and calcium-transporting ATPase indicates that these genes may play important roles in the grooming and hygienic behaviors.

Many studies have suggested that bees with the greatest olfactory sensitivity generally initiate hygienic behavior first because they can detect and accurately discriminate between abnormal and normal broods at low stimulus intensities (Martin et al., 2001; Swanson et al., 2009). Differences in olfaction may account for bee tolerance to Varroa as well as increased grooming and hygienic behavior, both of which are known to be involved in Varroa tolerance (Navajas et al., 2008). The differential expression of 3 members of the odorant binding protein (obp) family (obp 4, obp 17, and obp 18) according to bee genotype is very important considering that in A. cerana, olfaction may play an important role in the detection of Varroa-infested cells.

The recognition and discrimination of thousands of odorous compounds is mediated by olfactory sensory neurons. In insects, chemosensory neurons are surrounded by an aqueous milieu that acts as a barrier for volatile primarily lipophilic molecules. Thus, many airborne molecules, such as hydrophobic odorants and pheromones, must first be recognized by a specialized class of proteins that facilitate their delivery to olfactory receptors. It is widely accepted that this function is provided in insects by obps (Deyu and Leal, 2002). As such, upregulation of the 3 obps may support the bees' strong ability to detect Varroa. Among the 3 obps, only obp 4 was expressed exclusively in the antennae of adult A. mellifera bees, while obp 17 and obp 18 were ubiquitously expressed in all adult body parts (Forêt and Maleszka, 2006), suggesting that $o b p 17$ and $o b p 18$ are also involved in other physiological functions and that $o b p 4$ may be the most important gene in the sensitive olfactory sensation of $A$. cerana. Furthermore, the Down syndrome cell adhesion molecule gene, which is also involved in olfaction (Hummel et al., 2001), was upregulated in the Con2 colony. The differential expression of this group of genes is very important as olfaction plays an important role in the detection of Varroa-infested cells.

Behavior is influenced by both heritable and environmental factors, sometimes via massive changes in brain transcriptomes (Robinson, 2004). Behavior-specific neurogenomic states can be inferred from the coordinated action of transcription factors and their predicted target genes. Two transcription factors, cAMP-responsive element-binding protein-like 2-like and Mblk-1, were significantly upregulated in the Con2 colony. The identification of possible target genes for Mblk-1 and cAMP-responsive element-binding protein as well as their biological functions may provide important clues to the molecular basis of Varroa resistance mechanisms in A. cerana. 
Mblk-1, a transcription factor involved in the neuronal circuits of mushroom bodies (MBs), is preferentially expressed in the mb of the honeybee brain (Takeuchi et al., 2001; Park et al., 2003). Grooming and hygienic behaviors are social behaviors in honeybees that may require complex processing of sensory information. However, little is known regarding the molecular basis of their highly advanced behavior. MBs are thought to be involved in sensory integration, learning, and memory in insects (Groh et al., 2006). Honeybee MBs are well-developed compared with those of other insects (McQuillan et al., 2012), suggesting that MB function is closely associated with advanced honeybee behaviors. Because Mblk-1 and its target genes are preferentially expressed in the $\mathrm{MB}$, they may play an important role in grooming and hygienic behaviors. The cAMP-responsive element-binding protein is a cAMPresponsive transcription factor that has been implicated in the activation of protein synthesis required for long-term facilitation, a cellular model of memory (Warburton et al., 2005). The cAMP signaling system plays critical roles in producing long-term memories (LTM), or longterm synaptic plasticity considered to underlie LTM formation, in insects. cAMP-responsive element-binding protein activation leads to long-term protein synthesis-dependent synaptic plasticity underlying LTM formation (Chen et al., 2012). These findings suggest that LTM, such as olfactory learning, formed when eastern honeybees developed Varroa resistance (Dupuis et al., 2012). Accordingly, we hypothesize that $A$. cerana forms an LTM of specific $V$. destructor odors so that they can easily recognize mites during the next infestation.

$V$. destructor is the greatest threat to the honeybee, A. mellifera, worldwide, where it rarely causes serious harm to its native host, the eastern honeybee, A. cerana. The mechanisms underlying the genetic resistance of $A$. cerana to Varroa mites remain unclear. In this study, we identified genes involved in resistance to Varroa including unigenes related to the movement of skeletal muscle, olfactory sensitivity, and transcription factors. This finding confirmed that hygienic behavior and grooming behavior may play important roles in the resistance to Varroa. However, regulation nets among these genes should be investigated in future studies.

\section{Conflict of interest}

The authors declare that they have no conflicts of interest.

\section{ACKNOWLEDGMENTS}

Research supported by the Grant from the National Natural Science Foundation of China (\#31372382), the Earmarked Fund for Modern Agro-Industry Technology Research System from the Ministry of Agriculture of China (\#CARS-45-SYZ6), and the Priority Academic Program Development of Jiangsu Higher Education Institutions.

\section{Supplementary material}

\section{REFERENCES}

Annoscia D, Del Piccolo F and Nazzi F (2012). How does the mite Varroa destructor kill the honeybee Apis mellifera? Alteration of cuticular hydrocarbons and water loss in infested honeybees. J. Insect Physiol. 58: 1548-1555.

Behrens D, Huang Q, Geßner C, Rosenkranz P, et al. (2012). Three QTL in the honey bee Apis mellifera L. suppress reproduction of the parasitic mite Varroa destructor. Ecol. Evol. 1: 451-458. 
Benjamini Y and Yekutieli D (2001). The control of the false discovery rate in multiple testing under dependency. Ann. Stat. 29: 1165-1188.

Bienefeld K, Zautke F, Pronin D and Mazedd A (1999). Recording the proportion of damaged Varroa jacobsoni in the debris of honey bee colonies (Apis mellifera). Apidologie 30: 249-256.

Boecking O (1992). Removal behavior of Apis mellifera towards sealed brood cells infested with Varroa jacobsoni: techniques, extent and efficacity. Apidologie 23: 371-373.

Chen CC, Wu JK, Lin HW, Pai TP, et al. (2012). Visualizing long-term memory formation in two neurons of the Drosophila brain. Science 335: 678-685.

Cornman RS, Tarpy DR, Chen Y, Jeffreys L, et al. (2012). Pathogen webs in collapsing honey bee colonies. PLoS One 7: e43562.

Damiani N, Gende LB, Bailac P, Marcangeli JA, et al. (2009). Acaricidal and insecticidal activity of essential oils on Varroa destructor (Acari: Varroidae) and Apis mellifera (Hymenoptera: Apidae). Parasitol. Res. 106: 145-152.

Deyu Z and Leal W (2002). Conformational isomers of insect odorant-binding proteins. Arch. Biochem. Biophys. 397 : 99-105.

Dupuis J, Louis T, Gauthier M and Raymond V (2012). Insights from honeybee (Apis mellifera) and fly (Drosophila melanogaster) nicotinic acetylcholine receptors: from genes to behavioral functions. Neurosci. Biobehav. Rev. 36 : 1553-1564.

Forêt $\mathrm{S}$ and Maleszka R (2006). Function and evolution of a gene family encoding odorant binding-like proteins in a social insect, the honey bee (Apis mellifera). Genome Res. 16: 1404-1413.

Franck P, Coussy H, Le Conte Y, Solignac M, et al. (1999). Microsatellite analysis of sperm mixture in honeybee. Insect Mol. Biol. 8: 419-421.

Grabherr M and Haas B (2011). Full-length transcriptome assembly from RNA-Seq data without a reference genome. Nat. Biotechnol. 29: 644-652.

Groh C, Ahrens D and Rossler W (2006). Environment- and age-dependent plasticity of synaptic complexes in the mushroom bodies of honeybee queens. Brain Behav. Evol. 68: 1-14.

Herranz R, Mateos J and Marco R (2005). Diversification and independent evolution of troponin C genes in insects. $J$. Mol. Evol. 60: 31-44.

Highfield AC, El Nagar A, Mackinder LC, Noël LM, et al. (2009). Deformed wing virus implicated in overwintering honeybee colony losses. Appl. Environ. Microbiol. 75: 7212-7220.

Hummel T, Vasconcelos M, Clemens J, Fishilevich Y, et al. (2001). Axonal targeting of olfactory receptor neurons in Drosophila is controlled by Dscam. Neuron 37: 221-231.

Kanga LH, Adamczyk J, Patt J, Gracia C, et al. (2010). Development of a user-friendly delivery method for the fungus Metarhizium anisopliae to control the ectoparasitic mite Varroa destructor in honey bee, Apis mellifera, colonies. Exp. Appl. Acarol. 52: 327-342.

Karazafiris E, Tananaki C, Menkissoglu-Spiroudi U and Thrasyvoulou A (2008). Residue distribution of the acaricide coumaphos in honey following application of a new slow-release formulation. Pest Manag. Sci. 64: 165-171.

Martin C, Provost E, Roux M, Bruchou C, et al. (2001). Resistance of the honey bee, Apis mellifera to the acarian parasite Varroa destructor: behavioural and electroantennographic data. Physiol. Entomol. 26: 362-370.

McQuillan HJ, Nakagawa S and Mercer AR (2012). Mushroom bodies of the honeybee brain show cell population-specific plasticity in expression of amine-receptor genes. Learn. Mem. 19: 151-158.

Mortazavi A and Williams B (2008). Mapping and quantifying mammalian transcriptomes by RNA-Seq. Nat. Methods 5: 621-628.

Navajas M, Migeon A, Alaux C, Martin-Magniette M, et al. (2008). Differential gene expression of the honey bee Apis mellifera associated with Varroa destructor infection. BMC Genomics 9: 301.

O'Connell K, Gannon J, Doran P and Ohlendieck K (2008). Reduced expression of sarcalumenin and related $\mathrm{Ca}^{2+}$ -regulatory proteins in aged rat skeletal muscle. Exp. Gerontol. 43: 958-961.

Oldroyd BP (1999). Coevolution while you wait: Varroa jacobsoni, a new parasite of western honeybees. Trends Ecol. Evol. 14: 312-315.

Park JM, Kunieda T and Kubo T (2003). The activity of Mblk-1, a mushroom body-selective transcription factor from the honeybee, is modulated by the ras/MAPK pathway. J. Biol. Chem. 278: 18689-18694.

Parker R, Guarna MM, Melathopoulos AP, Moon KM, et al. (2012). Correlation of proteome-wide changes with social immunity behaviors provides insight into resistance to the parasitic mite, Varroa destructor, in the honey bee (Apis mellifera). Genome Biol. 13: R81.

Prisco GD, Zhang X, Pennacchio F, Caprio E, et al. (2012). Dynamics of persistent and acute deformed wing virus infections in honey bees, Apis mellifera. Viruses 3: 2425-2441.

Rath W and Drescher W (1990). Response of Apis cerana Fabr towards brood infested with Varroa jacobsoni Oud and 
infestation rate of colonies in Thailand. Apidologie 21: 311-321.

Rinderer T, Harris J, Hunt G and Guzman L (2010). Breeding for resistance to Varroa destructor in North America. Apidologie 41: 409-424.

Robinson GE (2004). Genomics. Beyond nature and nurture. Science 304: 397-399.

Rosenkranz P, Fries I, Boecking O and Stürmer M (1997). Damaged Varroa mites in the debris of honey bee (Apis mellifera L.) colonies with and without hatching brood. Apidologie 28: 427-437.

Rosenkranz P, Aumeier P and Ziegelmann B (2009). Biology and control of Varroa destructor. J. Invertebr. Pathol. 103 (Suppl 1): S96-S119.

Sammataro D, Gerson U and Needham G (2000). Parasitic mites of honey bees: life history, implications, and impact. Annu. Rev. Entomol. 45: 519-548.

Sørensen TL, Møller JV and Nissen P (2004). Phosphoryl transfer and calcium ion occlusion in the calcium pump. Science 304: 1672-1675.

Swanson JA, Torto B, Kells SA, Mesce KA, et al. (2009). Odorants that induce hygienic behavior in honeybees: identification of volatile compounds in chalkbrood-infected honeybee larvae. J. Chem. Ecol. 35: 1108-1116.

Takeuchi H, Kage E, Sawata M, Kamikouchi A, et al. (2001). Identification of a novel gene, Mblk-1, that encodes a putative transcription factor expressed preferentially in the large-type Kenyon cells of the honeybee brain. Insect Mol. Biol. 10: 487-494.

Tan K, Yu Y and Zhang X (2002). Resistance of mites va rroa jacobsoni of Apis cerana colonies. Apiculture China 53: $10-12$.

Tsuruda JM, Harris JW, Bourgeois L, Danka RG, et al. (2012). High-resolution linkage analyses to identify genes that influence Varroa sensitive hygiene behavior in honey bees. PLoS One 7: e48276.

Warburton EC, Glover CP, Massey PV, Wan H, et al. (2005). cAMP responsive element-binding protein phosphorylation is necessary for perirhinal long-term potentiation and recognition memory. J. Neurosci. 25: 6296-6303. 